\title{
Investigations of a Possible Role of SNPs in KAII Gene on Its Down-Regulation in Breast Cancer
}

\author{
Haitham Kussaibi ${ }^{1 *}$, Khaled R. Alkharsah ${ }^{2}$
}

\begin{abstract}
Objective: KAI1 (CD82) is a metastasis suppressor gene known to be down-regulated in carcinomas of breast, prostate and many other organs. The mechanism of KAI1 down-regulation is complex and not well understood. Here, we investigate the role of 8 SNPs (not previously studied) in KAII gene that could influence its expression in tumor tissue samples of breast cancer patients from the Eastern province of Saudi Arabia. Methods: Single nucleotide polymorphisms (SNPs) in KAII gene were selected from the NCBI website (dbSNP) and were then filtered for those SNPs causing stop codon mutations (rs139889503 and rs150533529) or nonsynonymous mutation in the 5'-UTR (rs11541048, rs77359459, rs115500759, rs182579675, rs200238062, and rs372733853). SNPs genotyping was performed using TaqMan SNP Genotyping Assay and the results were correlated with KAI1 protein expression profile by immunohistochemistry (IHC) on formalin-fixed paraffin-embedded (FFPE) samples of breast cancer and control none-neoplastic tissues. Results: KAIl expression by IHC was observed in all none-neoplastic breast tissue samples and only in 35\% out of the 59 breast cancer tissue samples. None of the samples was homozygous for the stop codon allele A in the SNP rs139889503 or allele T in the SNP rs150533529. The SNPs in the 5-UTR, rs11541048, rs115500759, and rs182579675, were only present in the homozygous state for the $\mathrm{G}$ and $\mathrm{C}$ alleles respectively in both cancer and control samples. The other SNPs in the 5'-UTR (rs77359459, rs200238062, and rs372733853) had no significant difference in the allele distribution between KAI1 expressing or none-expressing tissue samples. Conclusion: Our findings showed no significant effect of the studied SNPs on down-regulation of KAII expression.
\end{abstract}

Keywords: KAI1 down-regulation- KAI1 gene loss of function- SNPs in KAI1 gene

Asian Pac J Cancer Prev, 21 (9), 2549-2553

\section{Introduction}

Breast cancer is a common cancer in the Eastern Province of Saudi Arabia with a high mortality rate which is mostly associated with its dissemination. Mechanism of cancer dissemination, in general, is mediated by complex effects of promoting genes and suppressor genes (Iiizumi et al., 2007). KAI1 (CD82) is a well-known metastasis suppressor trans-membrane glycoprotein. KAI1 prevents metastasis by inhibiting cancer cells mobility and infiltration (Liu and Zhang, 2006). To achieve its function, KAI1 protein interacts with other cell membrane proteins such as adhesion proteins, growth factor receptor (GFR), and signaling pathways (Bienstock and Barrett, 2001; Bari et al., 2009). Different types of cancers showed KAII loss of expression especially those in advanced stages (Christgen et al., 2009; Malik et al., 2009a; Malik et al., 2009b; Malik et al., 2009c; Mooez et al., 2011; Shiwu et al., 2012; Scarpino et al., 2013; Zhang et al., 2013). The mechanism behind down-regulation of $K A I 1$ expression during cancer development is complex, mediated by transcription control, alternative splicing, and even post-translational remodeling (Tonoli and Barrett, 2005; Tsai et al., 2007; Lee et al., 2011). Only a few studies investigated gene mutation, loss of heterozygosity $(\mathrm{LOH})$, or promoter hypermethylation as a cause of KAI1 down-regulation (Gao et al., 2003; Marreiros et al., 2003). One of the early studies revealed that neither mutation (on 10 prostatic cancer samples) nor allelic loss (on 34 prostatic cancer samples and 12 metastatic lymph nodes) is the cause of KAI1 gene down-regulation (Dong et al., 1995). A similar observation was made in another study (Miyazaki et al. 2000). Further studies also revealed that epigenetic changes such as methylation of promoter region is not essential to KAII loss of expression (Jackson et al., 2000; Sekita et al., 2001). A later report also showed that loss of KAI1 expression is not related to mutation, $\mathrm{LOH}$, epigenetic regulation of the promoter, or p53 mutation (Uzawa et al., 2002). On the other hand, a study on 52 ovarian carcinomas found a missense alteration at codon 241 (ATC to GTC), which causes valine to isoleucine substitution in the peptide sequence but it occurs in normal

${ }^{1}$ Department of Pathology, College of Medicine, Imam Abdulrahman Bin Faisal University (IAU), Dammam, Saudi Arabia. ${ }^{2}$ Department of Microbiology, College of Medicine, Imam Abdulrahman Bin Faisal University (IAU), Dammam, Saudi Arabia. *For Correspondence: hkussaibi@iau.edu.sa 
tissues in addition to tumor cells (Liu et al., 2001). One more study discovered a significant correlation between a SNP on exons 3 (-29166 C>T) and the development of colorectal cancer of high grade and advanced stage (Ma et al., 2013).

To our knowledge, after a thorough literature review, no previous study was done on the relationship between the SNPs (selected in our study) and the expression level of KAI1. We aim here to investigate the possibility of a significant association between these selected SNPs, and the $K A I 1$ expression, which could be if proved, a possible mechanism of KAII loss of expression in breast cancers.

\section{Materials and Methods}

\section{Sample collection}

Fifty-nine formalin-fixed paraffin-embedded (FFPE) tissue samples of breast cancer in addition to 20 FFPE none-neoplastic breast tissue samples (controls) were collected from the archive of the pathology department at King Fahd Hospital of the University (KFHU). All hematoxylin and eosin slides from tumor and control samples have been examined and verified by a breast pathologist. This study received an ethical approval from the Institutional Review Board (IRB) at Imam Abdulrahman Bin Faisal University (IRB number: IRB-2015-01-095).

\section{Immunohistochemistry study (IHC)}

Immunohistochemical KAI1 expression profile of cancer and control samples in the study, has been retrieved from our previous study (Kussaibi et al., 2019) which has been performed on FFPE tumor and control samples' paraffin sections (3-4 $\mu$ m thick), on Ventana Ultraview Autostainer using Ventana DAB detection universal kit (Ventana, Tucson, AZ, USA) following the standard protocol with further optimization, using as primary antibody (anti-CD82 TS82b, Abcam, Cambridge, UK). The antibody expression level of each sample was classified into: partial, diffuse or no expression.

\section{DNA extraction}

DNA was extracted from two 10-micrometer tissue sections using the FFPE DNA purification kit (Norgen Biotek, Ontario, Canada) according to the manufacturer's instructions. The tissue sections were first deparaffinized using 350ul of Qiagen deparaffinization solution for three minutes (Qiagen, Hilden, Germany) followed by $1 \mathrm{ml}$ of Xylene for 2 minutes. DNA quality and concentration were assessed using NanoDrop2000 (Life Technologies, California, USA).

\section{SNP selection}

After screening the KAI1 gene for SNP using the NCBI website, 3594 SNP were identified in homosapiens KAIl gene. All intronic and synonymous SNPs were excluded. The remaining SNPs were selected if they cause stop codon or if they were nonsynonymous and were located in the 5'-untranslated region. The following SNPs were predicted to cause stop or nonsense mutation: rs139889503, rs150533529, rs11541048, rs77359459, rs115500759, rs182579675, rs200238062, rs372733853.

\section{SNP genotyping}

Genotyping of the KAI1 SNPs was performed by TaqMan assay (Thermo Fisher Scientific, California, USA). The following SNPs were available in the company's database as validated assays; rs139889503, rs 150533529, rs 11541048, rs372733853. The other SNPs were ordered as an assay on demand. A twenty-microliter reaction volume containing specific primers and probes for each SNP separately was run on the 7500 Fast real-time PCR thermocycler (ABI, California, USA) according to the manufacturer's instructions. The software ABI 7500 was used for the endpoint allele calling.

\section{Data analysis}

Data were collected in an Excel sheet and be prepared for statistical analysis by IBM SPSS software v.24. Pearson's correlation coefficient (2-tailed) has been performed to measure the strength of the association between each of the mentioned SNPs and the level of KAII expression by IHC. Significant correlation was considered at $\mathrm{P}<0.050$.

\section{Results}

The expression of KAII by IHC

In a previous study, we investigated the expression level of KAII by immunohistochemistry from breast cancer tissue and none-neoplastic breast tissues using anti-CD82 TS82B (Kussaibi et al., 2019). Out of 59 tumor samples included in the current study, 35\% $(n=22 / 59)$ showed expression of KAI1 protein (partial or diffuse). The remaining $65 \%(n=37 / 59)$ showed loss of KAII expression. All the 20 control samples showed expression of KAII.

\section{Genotyping of the selected SNPs}

We searched the dbSNP database for SNPs in the gene coding region for $K A I 1$. Over three thousand SNPs were identified in the homosapiens KAII gene. We have then excluded all SNPs that are intronic or produce synonymous mutations. The remaining SNPs were further refined to SNPs which produce a stop codon (rs139889503 and rs 150533529) or if they were nonsynonymous mutations and were in the 5'-untranslated region (Table 1). None of the patients or controls in the study was homozygous for the stop-coding nucleotide (A) in the SNP rs139889503. Only one tumor patient was heterozygous for this SNP and the expression of KAI1 was not affected. Similarly, none of the patients or controls in the study was homozygous for the stop-coding nucleotide (T) in the second SNP rs 150533529 . However, 4 cases, including two normal controls, were heterozygous for this SNP (Table 1). The normal tissue showed expression of KAII in the two cases. While in the tumor samples, KAI1 was expressed in 50\% $(n=1 / 2)$ of the cases.

We also have looked for SNPs causing non-synonymous mutations in the 5'-UTR of KAI1 expecting them to affect KAI1 expression and identified six non-synonymous SNPs (Table 1). All the patient's samples and control samples 
DOI: 10.31557/APJCP.2020.21.9.2549

Investigations of a Possible Role of SNPs in KAII gene on its Down-Regulation in Breast Cancer

Table 1. Genotypes of KAI1 SNPs and Their Frequency among KAI1 Expressing and NoneExpressing Tissue Samples in the Study Population

\begin{tabular}{|c|c|c|c|c|c|c|c|}
\hline SNP & Genotype & $\begin{array}{c}\text { KAI1 } \\
\text { expressed }\end{array}$ & $\begin{array}{l}\text { KAI1 Not } \\
\text { expressed }\end{array}$ & $P$-value & $\begin{array}{c}\text { Functional } \\
\text { Consequence }\end{array}$ & Validation & MAF \\
\hline \multirow[t]{3}{*}{ rs139889503 } & $\mathrm{CC}$ & 23 & 35 & 0.437 & stop gained & by cluster & $A=0.000016$ \\
\hline & $\mathrm{AC}$ & 1 & 0 & & & & \\
\hline & AA & 0 & 0 & & & & \\
\hline \multirow[t]{3}{*}{ rs150533529 } & $\mathrm{CC}$ & 21 & 34 & 0.107 & stop gained & by cluster & $\mathrm{T}=0.000074$ \\
\hline & $\mathrm{CT}$ & 3 & 1 & & & & \\
\hline & $\mathrm{TT}$ & 0 & 0 & & & & \\
\hline \multirow[t]{2}{*}{ rs11541048 } & GG & 24 & 35 & - & 5'-UTR variant & by cluster & $\mathrm{A}=0.00003$ \\
\hline & $\mathrm{AA} / \mathrm{AG}$ & 0 & 0 & & & & \\
\hline \multirow[t]{3}{*}{ rs 77359459} & GG & 22 & 32 & 0.974 & 5'-UTR variant & by $1000 \mathrm{G}$ & $\mathrm{C}=0.0308$ \\
\hline & GA & 2 & 3 & & & by cluster & \\
\hline & AA & 0 & 0 & & & & \\
\hline \multirow[t]{2}{*}{ rs115500759 } & $\mathrm{CC}$ & 24 & 35 & 0.437 & 5'-UTR variant & by $1000 \mathrm{G}$ & $\mathrm{T}=0.0056$ \\
\hline & $\mathrm{CT} / \mathrm{TT}$ & 0 & 0 & & & $\begin{array}{l}\text { by cluster } \\
\text { by frequency }\end{array}$ & \\
\hline \multirow[t]{2}{*}{ rs182579675 } & $\mathrm{CC}$ & 24 & 35 & - & 5'-UTR variant & by $1000 \mathrm{G}$ & $\mathrm{T}=0.0002$ \\
\hline & $\mathrm{CT} / \mathrm{TT}$ & 0 & 0 & & & & \\
\hline \multirow[t]{3}{*}{ rs200238062 } & GG & 22 & 34 & 0.638 & 5'-UTR variant & by $1000 \mathrm{G}$ & $\mathrm{T}=0.0002$ \\
\hline & GA & 2 & 1 & & by cluster & & \\
\hline & AA & 0 & 0 & & & & \\
\hline \multirow[t]{3}{*}{ rs 372733853} & $\mathrm{TT}$ & 24 & 34 & 0.453 & 5'-UTR variant & no info & no info \\
\hline & $\mathrm{TC}$ & 0 & 1 & & & & \\
\hline & $\mathrm{CC}$ & 0 & 0 & & & & \\
\hline
\end{tabular}

were homozygous for the SNP rs182579675. Other SNPs were also not found in the homozygous state for the minor allele and there was no significant effect on the expression of KAII in the heterozygous state. Most of tumor and control samples showed almost the same SNP profile with no significant association with KAII expression status (Table 1).

\section{Discussion}

KAI1 is a transmembrane glycoprotein that inhibits tumor metastasis by inducing senescence of tumor migrating cells at the endothelial surface through interaction with Duffy antigen chemokine receptor (Bandyopadhyay et al., 2006). Therefore, its expression is usually downregulated in many tumors particularly in advance stages. Understanding the mechanism of $K A I 1$ loss of expression in advanced tumor is crucial to understand the mechanism of metastasis inhibition. A polymorphism in the KAIl gene or its regulatory region that may accumulate during the tumor growth could affect its expression and lead to reduced translation or complete loss of expression.

Our findings showed that most of tumor and control samples in the study showed almost the same SNP profile with no significant association with KAI1 expression status in breast cancer tissues. We investigated the effect of SNPs that may produce stop codon and lead to truncation of the protein on KAII expression. None of the samples was homozygous for the allele that produces stop mutation. However, four cases, including two normal controls, were heterozygous for these SNPs. The normal tissue showed expression of KAIl in the two cases. While in the tumor samples, KAI1 was expressed in $50 \%(n=1 / 2)$ of the cases. This indicates that the heterozygous state of these two SNPs does not affect KAII expression.

Similarly, no effect was observed for the nonsynonymous SNPs in the 5' -untranslated region (5'-UTR) of the KAIl gene on its expression. The 5'-UTR is an untranslated sequence in an mRNA directly upstream of the start codon, which plays an important role in the regulation of protein expression by influencing the translation of its transcript.

These SNPs were not previously studied, and no data was available about them in the literature other than the databases. A study investigating SNP in multiple metastasis inhibitor genes including KAI1 did not find a significant association between particular SNP and risk of breast cancer but rather found an effect of multiple variants in several genes including KAIl on the risk of breast cancer when there is lymph nodes involvement (Roberts et al., 2017). Therefore, there could be no one SNPs that by itself affects the KAII expression but rather a combined effect of multiple SNPs.

A study on patients with head and neck cancer concluded that germline mutation in KAII gene could be a less frequent event (Nazir et al., 2011).

The small sample size could be a limitation in our study. Therefore, further studies are required to understand KAI1 down-regulation in tumor tissue which could be not 
only at the germline level but also at the transcriptional or translational level (epigenetic regulation) (Lee et al., 2017).

In conclusion, our findings did not reveal significant association between the studied SNPs and the KAII expression in breast cancer, which again makes mutations a less likely mechanism of KAI1 loss of expression.

\section{Acknowledgments}

The authors are grateful to Mrs. Maria Rosa, Histo Lab specialist at King Fahd Hospital of the University and Mrs. Eman Alsulami, for their valuable technical contribution. This work was funded By Deanship for Scientific Research at Imam Abdulrahman Bin Faisal University (Project number 2015192).

\section{Ethics approval and consent}

- This study received an ethical approval from the Institutional Review Board (IRB) at Imam Abdulrahman Bin Faisal University (IRB number: IRB-2015-01-095).

- Due to the use of archival material of anonymous nature that does not reveal patients' identity and because patients are lost to follow-up, informed patient consent was waived.

\section{Availability of data and material}

The data file related to this study is available from the corresponding author on reasonable request.

\section{Funding}

This work was funded By Deanship for Scientific Research at Imam Abdulrahman Bin Faisal University (Project number 2015192)

\section{References}

Bandyopadhyay S, Zhan R, Chaudhuri A, et al (2006). Interaction of KAI1 on tumor cells with DARC on vascular endothelium leads to metastasis suppression. Nat Med, 12, 933-8.

Bari R, Zhang YH, Zhang F, et al (2009). Transmembrane interactions are needed for KAI1/CD82-mediated suppression of cancer invasion and metastasis. Am J Pathol, 174, 647-60.

Bienstock RJ, Barrett JC (2001). KAI1, a prostate metastasis suppressor: prediction of solvated structure and interactions with binding partners; integrins, cadherins, and cell-surface receptor proteins. Mol Carcinog, 32, 139-53.

Christgen M, Christgen H, Heil C, et al (2009). Expression of KAI1/CD82 in distant metastases from estrogen receptor-negative breast cancer. Cancer Sci, 100, 1767-71.

Dong JT, Lamb PW, Rinker-Schaeffer CW, et al (1995). KAI1, a metastasis suppressor gene for prostate cancer on human chromosome 11p11.2. Science, 268, 884-6.

Gao AC, Lou W, Dong JT, et al (2003). Defining regulatory elements in the human KAI1 (CD 82) metastasis suppressor gene. Prostate, 57, 256-60.

Iiizumi M, Bandyopadhyay S, Watabe K (2007). Interaction of Duffy antigen receptor for chemokines and KAI1: a critical step in metastasis suppression. Cancer Res, 67, 1411-4.

Jackson P, Millar D, Kingsley E, et al (2000). Methylation of a $\mathrm{CpG}$ island within the promoter region of the KAI1 metastasis suppressor gene is not responsible for downregulation of KAI1 expression in invasive cancers or cancer cell lines. Cancer Lett, 157, 169-76.

Kussaibi H, Alkharsah KR, Altamimi D, et al (2019). Alternative splicing is an important mechanism behind KAI1 loss of function in breast cancer patients from Saudi Arabia. Breast Cancer Res Treat, 173, 87-91.

Lee HA, Park I, Byun HJ, et al (2011). Metastasis suppressor KAI1/CD82 attenuates the matrix adhesion of human prostate cancer cells by suppressing fibronectin expression and beta1 integrin activation. Cell Physiol Biochem, 27, 575-86.

Lee J, Lee MS, Jeoung DI, Kim YM, Lee H (2017). Promoter CpG-Site Methylation of the KAI1 Metastasis Suppressor Gene Contributes to Its Epigenetic Repression in Prostate Cancer. Prostate, 77, 350-60.

Liu FS, Chen JT, Dong JT, et al (2001). KAI1 metastasis suppressor gene is frequently down-regulated in cervical carcinoma. Am J Pathol, 159, 1629-34.

Liu WM, Zhang XA (2006). KAI1/CD82, a tumor metastasis suppressor. Cancer Lett, 240, 183-94.

Ma ZB, Li K, Wang J, Guo GH (2013). Role of KAI1/CD82 polymorphisms in colon cancer risk in Han Chinese population. Med Oncol, 30, 668.

Malik FA, Sanders AJ, Jiang WG (2009a). KAI-1/CD82, the molecule and clinical implication in cancer and cancer metastasis. Histol Histopathol, 24, 519-30.

Malik FA, Sanders AJ, Jones AD, Mansel RE, Jiang WG (2009b). Transcriptional and translational modulation of KAI1 expression in ductal carcinoma of the breast and the prognostic significance. Int J Mol Med, 23, 273-8.

Malik FA, Sanders AJ, Kayani MA, Jiang WG (2009c). Effect of expressional alteration of KAI1 on breast cancer cell growth, adhesion, migration and invasion. Cancer Genomics Proteomics, 6, 205-13.

Marreiros A, Czolij R, Yardley G, Crossley M, Jackson P (2003). Identification of regulatory regions within the KAI1 promoter: a role for binding of AP1, AP2 and p53. Gene, 302, 155-64.

Miyazaki T, Kato H, Shitara Y, et al (2000). Mutation and expression of the metastasis suppressor gene KAI1 in esophageal squamous cell carcinoma. Cancer, 89, 955-62.

Mooez S, Malik FA, Kayani MA, et al (2011). Expressional alterations and transcript isoforms of metastasis suppressor genes (KAI1 and KiSS1) in breast cancer patients. Asian Pac J Cancer Prev, 12, 2785-91.

Nazir M, Kayani MR, Malik FA, Masood N, Kayani MA(2011). Lack of germ line changes in KISS1 and KAI1 genes in sporadic head and neck cancer patients of Pakistani origin. Asian Pac J Cancer Prev, 12, 2767-71.

Roberts MR, Sucheston-Campbell LE, Zirpoli GR, et al (2017). Single nucleotide variants in metastasis-related genes are associated with breast cancer risk, by lymph node involvement and estrogen receptor status, in women with European and African ancestry. Mol Carcinog, 56, 1000-9.

Scarpino S, Duranti E, Giglio S, et al (2013). Papillary carcinoma of the thyroid: high expression of COX-2 and low expression of KAI-1/CD82 are associated with increased tumor invasiveness. Thyroid, 23, 1127-37.

Sekita N, Suzuki H, Ichikawa T, et al (2001). Epigenetic regulation of the KAI1 metastasis suppressor gene in human prostate cancer cell lines. Jpn J Cancer Res, 92, 947-51.

Shiwu WU, Lan Y, Wenqing S, Lei Z, Yisheng T (2012). Expression and clinical significance of CD82/KAI1 and E-cadherin in non-small cell lung cancer. Arch Iran Med, 15, 707-12.

Tonoli H, Barrett JC (2005). CD82 metastasis suppressor gene: 
a potential target for new therapeutics?. Trends Mol Med, 11, 563-70.

Tsai YC, Mendoza A, Mariano JM, et al (2007). The ubiquitin ligase gp 78 promotes sarcoma metastasis by targeting KAI1 for degradation. Nat Med, 13, 1504-9.

Uzawa K, Ono K, Suzuki H, et al (2002). High prevalence of decreased expression of KAI1 metastasis suppressor in human oral carcinogenesis. Clin Cancer Res, 8, 828-35.

Zhang BH, Liu W, Li L, et al (2013). KAI1/CD82 and MRP1/ CD9 serve as markers of infiltration, metastasis, and prognosis in laryngeal squamous cell carcinomas. Asian Pac J Cancer Prev, 14, 3521-6.

\section{c) (7) (8)}

This work is licensed under a Creative Commons AttributionNon Commercial 4.0 International License. 